\section{FRI0447 WORLDWIDE EXPERT AGREEMENT ON UPDATED EULAR/EUSTAR RECOMMENDATIONS FOR THE TREATMENT OF SYSTEMIC SCLEROSIS}

J. De Vries-Bouwstra ${ }^{1}$, Y. Allanore ${ }^{2}$, M. Matucci-Cerinic ${ }^{3}$, A. Balbir-Gurman ${ }^{4}$ ${ }^{1}$ Rheumatology, Leiden University Medical Center, Leiden, Netherlands; ${ }^{2}$ Rheumatology, Cochin Hospital, Paris, France; ${ }^{3}$ Experimental and Clinical Medicine, University of Florence, Florence, Italy; ${ }^{4} B$. Shine Rheumatology Unit Rappaport Faculty of Medicine- Rambam Health Care Campus, Haifa, Israel

Background: In 2017, updated EULAR/EUSTAR recommendations for treatment of Systemic sclerosis (SSc) were published. ${ }^{1}$ Implementation in clinical practice of these recommendations might be dependent on agreement and local drug availability.

Objectives: To evaluate worldwide agreement of the updated recommendations for treatment of SSc among SSc experts. To determine factors that might influence agreement.

Methods: An online survey was set out (June - September 2017), containing the 17 EULAR/EUSTAR updated recommendations. Levels of agreement were determined for each item on a 10 -point scale $(0=$ not at all; $10=$ completely agree). Local drug availability was assessed (yes/no). An e-mail containing a web link to the survey was sent out to international SSc-networks (EUSTAR, Scleroderma Clinica Trials Consortium, Australian Scleroderma Interest Groups, International Systemic Sclerosis Inception Cohort) and to 54 additional SSc-experts representing South-America and Asia. Levels of agreement were compared between subgroups stratified for EUSTAR membership, geographical area, drug availability, and years of experience.

Results: In total 269 responders participated, of whom $n=209$ completed each single item. The majority are rheumatologists $(n=200,83 \%)$, currently working in Europe $(n=185 ; 71 \%) ; 65 \%(n=156)$ are EUSTAR member, and have $>10$ years of experience in the SSc field $(n=150 ; 63 \%)$. Other geographical areas are also represented (Africa $n=2,1 \%$; Asia $n=18,7 \%$; Australia $n=13,5 \%$; North-America $n=27,10 \%$; South-America $n=17,6 \%)$. Mean level of agreement was high (8.0; standard deviation [SD, 2.5], with top-3 highest mean agreement for ACE-inhibitors for scleroderma renal crisis (9.2 [2.1]), blood pressure control in case of corticosteroids (9.0 [2.2]) and treatment with protonpump inhibitors to prevent reflux complications (9.0 [2.2]). Top 3 of lowest mean agreement included fluoxetine for Raynauds phenomenon (RP) (4.6[ 2.8]), hematopoietic stem cell transplantation (HSCT) for severe SSc (7.1 [2.9]) and methotrexate for skin involvement in early diffuse SSc (7.4[ 2.8]). When comparing Europe to other regions agreement differed significantly $(p<0.05)$ for the following recommendations: iloprost for RP, iloprost and bosentan for digital ulcers, methotrexate for early diffuse SSc, cyclophosphamide for SSc-related lung disease, and HSCT for severe SSc. Comparison of agreement between responders stratified for EUSTAR membership (yes/no), years of clinical experience ( $<0 r>10$ years) and local availability of the drug/treatment option (yes/no) showed that in case of iloprost, bosentan and HSCT availability of drug/treatment option contributed to differences in agreement. Neither EUSTAR membership nor years of experience could explain these differences.

Conclusions: In general, worldwide agreement on recently updated recommendations for treatment of SSc is high. Differences in agreement are partially explained by geographical area and local drug availability. Future evaluations should focus on implementation of published recommendations and take drug availability into account to further improve guideline-development.

REFERENCE:

[1] Ann Rheum Dis. 2017 Aug;76(8):1327.

Disclosure of Interest: None declared

DOI: 10.1136/annrheumdis-2018-eular.7132

\section{FRI0448 MDA5 ANTIBODY POSITIVE CLINICAL AMYOPATHIC DERMATOMYOSITIS (CADM): A SINGLE TERTIARY CENTRE CASE SERIES OF 13 PATIENTS}

K. Huang, K. Shojania, J. Yeung, A. Avina-Zubieta. Rheumatology, University of British Columbia, Vancouver, Canada

Background: Positive anti-melanoma differentiation-associated gene 5 (MDA5) antibody has been reported in $50 \%-73 \%$ of patients with clinical amyopathic dermatomyositis $(\mathrm{CADM})^{1}$. About half of MDA5 +CADM patients die of rapid progressive interstitial lung disease (RP-ILD), defined as worsening dyspnea within 1 month that required high-flow oxygen therapy or ventilator support in previously stable patients.

Objectives: This descriptive study aims to systematically characterise the clinical features, treatment and outcome of 13 cases of MDA5 +CADM patients at a single referral centre.

Methods: Myositis antibody testing became available at Vancouver Coastal Health, Canada since 2014. We retrospectively reviewed clinical presentations, laboratories, imaging studies, treatment and outcome of patients with MDA5 antibody. The data is presented descriptively.

Results: Total 13 cases ( 4 male and 9 female) of MDA5 +CADM were identified (2014-2017). Mean age was 49.9 \pm 11.5 . Ten were Asian descent ( 8 Chinese, 1 Philippine, and 1 Indo-Mauritian) and 3 Caucasians. All 13 patients had typical DM rash (heliotrope, Gottron's, mechanic's hands, periungual erythema). Cutaneous ulcers over the extensor surfaces of joints were seen in $7 / 13$ and palmar papules in $4 / 13$, both of which were characteristic for MDA5 +CADM. Only 3/13 patients had CK elevation with little muscle weakness; all others had normal CK and no detectable weakness.

ILD was present in all 13 patients. 7/13 (53.8\%) patients had RP-ILD and 4/13 $(30.7 \%)$ died within 3 months of onset of worsening dyspnea, despite steroid, cyclophosphamide, Rituximab, cyclosporine and IVIG; 3 survived after receiving lung transplant. $6 / 13(46.2 \%)$ patients had stable ILD and are still alive. 5/7 patients with RP-ILD (71.4\%) had coexisting Ro52 antibody whereas only $1 / 6$ patients with stable ILD (16.7\%) had Ro52. Of the 6 patients with stable ILD, one had worsening cutaneous ulceration and ILD that required IV cyclophosphamide; one had refractory dermatitis and recurrent pneumomediastinum eventually stabilised on rituximab; one responded to tacrolimus with improving respiratory symptoms; one had panniculitis refractory to IVIg, hydroxychloroquine, azathioprine and tofacitinib, and ultimately responded to IV cyclophosphamide; one had mild ILD but severe cutaneous disease who eventually agreed to rituximab, and the last patient went with traditional Chinese medicine and lost follow up. None had underlying malignancy.

Conclusions: In this case series of 13 patients with MDA5 +CADM, $54 \%$ were Chinese, a finding similar to previous reported higher incidence in Asians. It is important to recognise the characteristic cutaneous and pulmonary features of this entity early on as mortality is high. Refractory panniculitis can be found in MDA5 +CADM. All patients had ILD with 54\% RP-ILD and $31 \%$ mortality within 3 months. Coexisting Ro52 may be predictive of worse prognosis. Early intensive immunosuppression is instrumental for refractory cutaneous manifestation and/o progressive ILD. As lung transplantation became more available, three patients with RP-ILD in this series received lung transplant successfully and they were maintained on mycophenolate and tacrolimus after transplant.

\section{REFERENCE:}

[1] Sato S, et al. Autoantibodies to a 140-kd polypeptide, CADM-140, in Japanse patients with clinically amyopathic dermatomyositis. Arthritis Rheum 2005;52:1571-6.

Disclosure of Interest: None declared DOI: 10.1136/annrheumdis-2018-eular.1887

\section{FRI0449 OVERLAP MYOSITIS AND PRIMARY MYOSITIS: CLINICAL OUTCOMES AND PROGNOSIS}

L. Nuño ${ }^{1}$,B.E. Joven ${ }^{2}$, P.E. Carreira ${ }^{2}$, J. Martínez Barrio ${ }^{3}$, V. Maldonado-Romero ${ }^{4}$, C. Larena-Grijalba ${ }^{4}$,I. Llorente Cubas ${ }^{5}$,E.G. Tomero ${ }^{5}$, M.C. Barbadillo-Mateos ${ }^{6}$, P. García de la Peña Lefebvre ${ }^{7}$, L. Ruiz-Gutiérrez ${ }^{8}$, J.C. López-Robledillo ${ }^{8}$ H. Moruno-Cruz ${ }^{9}$, A. Pérez ${ }^{9}$, T. Cobo-lbáñez $^{10}$, R. Almodóvar González ${ }^{11}, M$. J. García-de Yébenes ${ }^{12}$, L. Lojo ${ }^{13}$,F.J. López-Longo ${ }^{3}$, on behalf of REMICAM study group. ${ }^{1}$ Rheumatology, H. La Paz; ${ }^{2}$ Rheumatology, H. Doce de Octubre;

${ }^{3}$ Rheumatology, H. Gregorio Marañón; ${ }^{4}$ Rheumatology, H. Ramón y Cajal;

${ }^{5}$ Rheumatology, H. La Princesa; ${ }^{6}$ Rheumatology, H. Puerta de Hierro;

${ }^{7}$ Rheumatology, H. Madrid Norte Sanchinarro; ${ }^{8}$ Rheumatology, H. Infantil Niño Jesús, Madrid; ${ }^{9}$ Rheumatology, H. Príncipe de Asturias, Alcalá de Henares; ${ }^{10}$ Rheumatology, H. Infanta Sofía, San Sebastián de los Reyes; ${ }^{11}$ Rheumatology, H. Fundación Alcorcón, Alcorcón; ${ }^{12}$ InMusc; ${ }^{13}$ Rheumatology, H. Infanta Leonor, Madrid, Spain

Background: Among the subtypes of idiopathic inflammatory myopathy (IIM), it has been recognised that primary polymyositis (PM) and primary dermatomyositis 